\title{
New lower bounds for Heilbronn numbers
}

\author{
Francesc Comellas, J. Luis A. Yebra* \\ Departament de Matemàtica Aplicada IV \\ Universitat Politècnica de Catalunya \\ Escola Politècnica Superior de Castelldefels \\ Av. del Canal Olímpic, s.n. \\ 08860 Castelldefels, Catalonia, Spain \\ \{comellas, yebra\}@mat.upc.es
}

Submitted: October 1, 2001; Accepted: February 2, 2002.

MR Subject Classifications: 52C35, 52A40, 51M25

\begin{abstract}
The $n$-th Heilbronn number, $H_{n}$, is the largest value such that $n$ points can be placed in the unit square in such a way that all possible triangles defined by any three of the points have area at least $H_{n}$. In this note we establish new bounds for the first Heilbronn numbers. These new values have been found by using a simple implementation of simulated annealing to obtain a first approximation and then optimizing the results by finding the nearest exact local maximum.
\end{abstract}

\section{Introduction}

Let $x_{1}, x_{2}, \ldots, x_{n}$ be $n$ points in the unit square. Denote by $A\left(x_{1}, x_{2}, \ldots, x_{n}\right)$ the smallest area of all the possible triangles induced by the $n$ points. H.A. Heilbronn (1908-1975) asked for the exact value, or for an approximation of $H_{n}=\max _{x_{1}, x_{2}, \ldots, x_{n}} A\left(x_{1}, x_{2}, \ldots, x_{n}\right)$ and conjectured that $H_{n}=O\left(1 / n^{2}\right)$. Roth published in 1951 [14] an upper bound $H_{n}=$ $O(1 / n \sqrt{\log \log n})$ and a construction from P. Erdös which shows that $H_{n}$ is not of lower order than $n^{-2}$, so that if the conjecture is true then it would be tight. The upper bound was improved in 1972 by W.M. Schmidt [19] and by K.F. Roth, who studied the problem extensively and published several paper between 1972 and 1976 [15, 16, 17, 18], with refinements on the bound. Finally, and considering probabilistic arguments, the conjecture was disproved by Kómlos, Pintz and Szemerédi [11, 12], by showing that, for large $n, n^{-2}(\log n) \ll H_{n} \ll n^{-\frac{8}{7}+\epsilon}$.

* Research supported by the Ministry of Science and Technology, Spain, and the European Regional Development Fund (ERDF) under project TIC-2001-2171. 
Recent approaches to the Heilbronn problem include an algorithm, provided in 1997 by C. Bertram-Kretzberg, T. Hofmeister, and H. Lefmann [3], which for a discretization of the problem finds a triangle with area $\log n / n^{2}$ for every fixed $n$; lower bounds on higher dimensional versions of the problem, produced in 1999 by G. Barequet [2] and the study of the average size of the triangles by T. Jiang, M. Li, and P. Vitányi [8, 9].

In addition, Golberg [7] started in 1972 the determination of optimal values and lower bounds for every $H_{n}$. Besides the trivial cases $H_{3}$ and $H_{4}$, Golberg showed constructions providing lower bounds for $n \leq 15$. A.W.M. Dress, Yang Lu, Zhang Jingzhong and Zeng Zhenbing in a series of preprints and papers $[20,21,22,6]$ proved that the value of $H_{6}$ given by Goldberg was optimal and provided also the optimal value for $H_{5}$. They also improved the lower bound for $H_{7}$.

In this note, and by using a simple implementation of simulated annealing and a further optimization procedure, we present new lower bounds for the first Heilbronn numbers for which no optimal value is known, namely $7 \leq n \leq 12$. We have no proof for the optimality of the new bounds, but the optimization method used gives us confidence that very likely these values are optimal.

\section{The optimization method}

The technique we use to obtain new constructions for the Heilbronn problem has two steps. First we use a computer implementation of simulated annealing (SA) to find a good configuration of points and then an analytical study to attain a close local maximum.

\subsection{The simulated annealing approach}

We use a standard implementation of simulated annealing $[1,10]$ based on the code described in Scientific American [4]. The cost function $f(i)$, for a given configuration $i$ of $n$ points, is the area of the smallest triangle and we try to maximize it. A geometrical cooling rate, $T_{k}=0.9 T_{k-1}$, is used. Typical values for the main parameters are: initial temperature $T_{0}=0.01$; number of temperature changes, $N_{\max }$, from 100 to 1000; maximum number of iterations for a given temperature runLimit $=1000 \mathrm{n}$; number of succesful changes accepted before a new temperature (and cycle of iterations) is attempted sucLimit $=100$.

Set $T_{0}, N_{\max }$, runLimit, sucLimit;

Initialize $n$ points randomly to obtain configuration $i$

For k from 1 to $N_{\max }$ do

Repeat

Generate configuration $j$ by changing one of the points of configuration $i$

$f(j)=$ area of the smallest triangle of $j$

If $f(j) \geq f(i)$

Then $i \leftarrow j$

Else 


$$
\text { If } e^{(f(i)-f(j)) / T_{k}}<\text { random }[0,1)
$$

Then $i \leftarrow j$

Until runLimit or sucLimit

$T \leftarrow 0.9 T$

End For

We run this implementation several hundred times for each number of points, saving the best solutions. This leads to quasi-optimal configurations of points that are equivalent under symmetries and such that there are several smallest triangles wich have almost the same area, providing a first estimate of the corresponding Heilbronn number.

\subsection{The local optimization procedure}

The above algorithm produces good approximations to local maxima. In every particular case we choose the best one and improve it to the closest local maximum. To verify that we attain a local maximum we use the following result that can be found in [5].

Theorem Let $f=\min \left\{f_{1}, f_{2}, \ldots, f_{l}\right\}$ where $f_{1}, f_{2}, \ldots, f_{l}$ are continuously differentiable functions of $t=\left(t_{1}, t_{2}, \ldots, t_{m}\right)$, and let $t^{*}$ be a point at which

$$
f_{1}\left(t^{*}\right)=f_{2}\left(t^{*}\right)=\cdots=f_{k+1}\left(t^{*}\right)<f_{k+2}\left(t^{*}\right) \leq \cdots \leq f_{l}\left(t^{*}\right), \quad 1 \leq k \leq m,
$$

holds for some $k$. A necessary condition for $t^{*}$ to be a local maximum is that the $k+1$ gradients $\nabla f_{1}\left(t^{*}\right), \nabla f_{2}\left(t^{*}\right), \ldots, \nabla f_{k+1}\left(t^{*}\right)$, are linearly dependent through a combination with nonnegative coefficients:

$$
c_{1} \nabla f_{1}\left(t^{*}\right)+c_{2} \nabla f_{2}\left(t^{*}\right)+\cdots+c_{k+1} \nabla f_{k+1}\left(t^{*}\right)=0, \quad c_{i} \geq 0 .
$$

Remark A point here corresponds to a concrete configuration of the Heilbronn problem. The linearity dependence condition provides $m-k$ equations that together with the $k$ equations $f_{1}\left(t^{*}\right)=f_{2}\left(t^{*}\right)=\cdots=f_{k+1}\left(t^{*}\right)$ leads to the points, among which the local maxima are to be found. Asking for the linear combination to have strictly positive coefficients (and not just nonnegative) assures that they are, in a certain sense, stationary points of $f$, see [5] for more details. The fulfilment of this condition together with the context of the situation serves to characterise the local maxima of $f$.

As an illustration of the local optimisation procedure, we detail here the $H_{8}$ and $H_{10}$ cases.

In the $H_{8}$ case the $\mathrm{SA}$ algorithm leads to a near optimum point where the smallest areas

$S_{1}=\frac{1}{2}(1-x) y, \quad S_{2}=\frac{1}{2}(1-2 x)(1-y)-\frac{1}{2}(1-x) z, \quad S_{3}=\frac{1}{2}(1-z) x \quad$ and $\quad S_{4}=\frac{1}{2}(z-x)$

have very close values. Equating them and solving for $x, y$ and $z$, we get the values

$$
x=\frac{5-\sqrt{13}}{6}=0.232408, \quad y=\frac{7-\sqrt{13}}{18}=0.18858, \quad z=\frac{7-\sqrt{13}}{9}=0.37761
$$


for which

$$
S_{1}=S_{2}=S_{3}=S_{4}=\frac{\sqrt{13}-1}{36}=0.072376 .
$$

Thus, $m=k=3$, and the linearity dependence condition, which trivially holds, is not required in order to find the sensible points. Since at the selected point this linear combination has positive coefficients:

$$
(48 \sqrt{13}-126) \nabla S_{1}+(19 \sqrt{13}-1) \nabla S_{2}+(41 \sqrt{13}+223) \nabla S_{3}+138 \nabla S_{4}=0,
$$

it is a stationary point, and indeed it is a local maximum.

In the $H_{10}$ case the $\mathrm{SA}$ algorithm leads to a near optimum point where the smallest areas

$$
S_{1}=\frac{1}{2} x(1-x-y), \quad S_{2}=\frac{1}{2} y(1-2 z)(1-y), \quad \text { and } \quad S_{3}=\frac{1}{2} z(1-x+y)-y
$$

have very close values (another one, $S_{4}=y(2 z-y)$, has a slightly larger value, but its inclusion among the smallest areas does not lead to a stationary point, failing to satisfy the positiveness condition on the linear dependence of the gradients.) Therefore, we have $m=3$ and $k=2$. We obtain two equations by equating the areas and a third one from the linear dependence of the gradients:

$$
\nabla S_{1}, \nabla S_{2} \quad \text { and } \quad \nabla S_{3} \quad \text { l.d. } \Rightarrow(1-2 x)(1-2 z)-y=0 .
$$

Solving the three equations for $x, y$ and $z$ we get the values

$$
\begin{gathered}
z=\frac{3}{4}-\frac{(63+8 \sqrt{62})^{1 / 3}}{12}-\frac{1}{12(63+8 \sqrt{62})^{1 / 3}}=0.315611 \\
x=\frac{z}{2}=0.157806, \quad y=(1-z)(1-2 z)=0.252387
\end{gathered}
$$

for which $S_{1}=S_{2}=S_{3}=\left(5 z^{2}-4 z^{3}\right) / 8=0.046537$. Again, at this point we obtain a linear combination with positive coefficients:

$$
\left(12 z^{2}-15 z+4\right) \nabla S_{1}+2(1-z) \nabla S_{2}+2 z \nabla S_{3}=0
$$

and the point is a local maximum. 


\section{Results for $H_{7}$ to $H_{12}$}

Below we list the coordinates of the points of the configurations obtained by applying our method to find new bounds for $H_{n}, 7 \leq n \leq 12$.

\section{Seven points}

Let $z$ be the root of $z^{3}+5 z^{2}-5 z+1=0, z=0.287258$. Then $H_{7} \geq \frac{1-14 z-2 z^{2}}{38}=0.083859$

\begin{tabular}{|c|c|}
\hline$-\frac{50}{19} z-\frac{17}{38} z^{2}+\frac{37}{38}$ & 0 \\
1 & 0 \\
0 & $z$ \\
$\frac{9}{19}+\frac{1}{19} z^{2}+\frac{7}{19} z$ & $z$ \\
$\frac{40}{19} z^{2}+\frac{223}{19} z-\frac{58}{19}$ & $-1+6 z+z^{2}$ \\
$\frac{58}{19} z-\frac{15}{19}+\frac{11}{19} z^{2}$ & 1 \\
1 & 1 \\
\hline
\end{tabular}

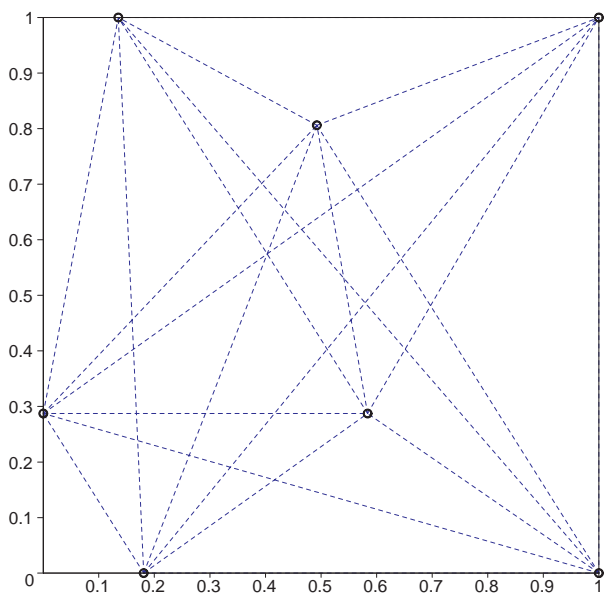

\section{Eight points}

$H_{8} \geq \frac{\sqrt{13}-1}{36}=0.072376$

\begin{tabular}{|c|c|}
\hline 0 & 0 \\
$\frac{1+\sqrt{13}}{6}$ & 0 \\
1 & $\frac{7-\sqrt{13}}{18}$ \\
$\frac{5-\sqrt{13}}{6}$ & $\frac{7-\sqrt{13}}{9}$ \\
$\frac{1+\sqrt{13}}{6}$ & $\frac{2+\sqrt{13}}{9}$ \\
0 & $\frac{11+\sqrt{13}}{18}$ \\
$\frac{5-\sqrt{13}}{6}$ & 1 \\
1 & 1 \\
\hline
\end{tabular}

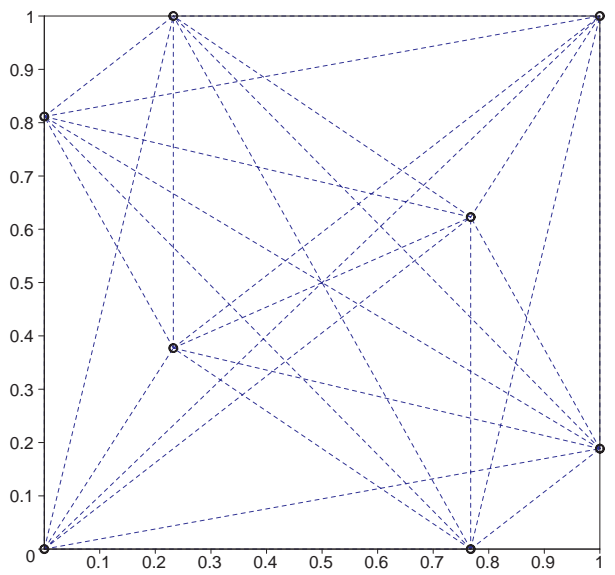


Nine points

$H_{9} \geq \frac{9 \sqrt{65}-55}{320}=0.054876$

\begin{tabular}{|c|c|}
\hline$\frac{10-\sqrt{65}}{10}$ & 0 \\
$\frac{25+\sqrt{65}}{40}$ & 0 \\
0 & $\frac{15-\sqrt{65}}{40}$ \\
1 & $\frac{15-\sqrt{65}}{40}$ \\
$\frac{15-\sqrt{65}}{20}$ & $\frac{5+\sqrt{65}}{20}$ \\
0 & $\frac{35+3 \sqrt{65}}{80}$ \\
1 & $\frac{\sqrt{65}}{10}$ \\
$\frac{45-3 \sqrt{65}}{80}$ & 1 \\
$\frac{25+\sqrt{65}}{40}$ & 1 \\
\hline
\end{tabular}

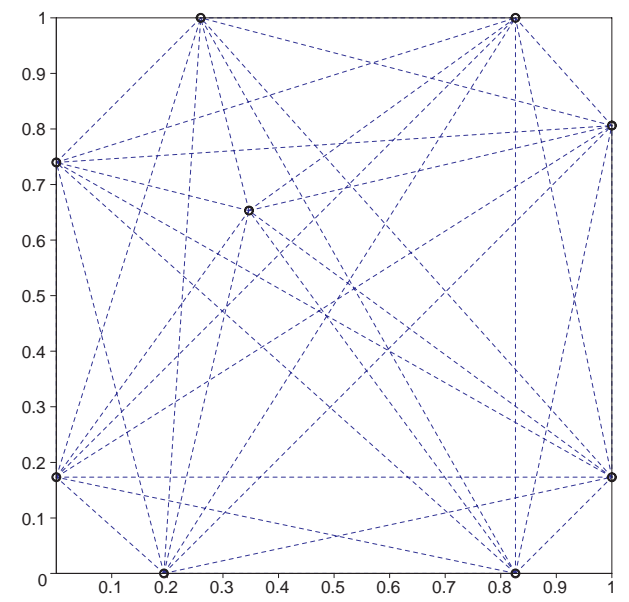

\section{Ten points}

With $z=-\frac{1}{12}(63+8 \sqrt{62})^{\frac{1}{3}}-\frac{1}{12}(63+8 \sqrt{62})^{-\frac{1}{3}}+\frac{3}{4}=0.315611$

and $x=\frac{z}{2}=0.157806$ and $y=1-3 z+2 z^{2}=0.252387 . H_{10} \geq \frac{5}{8} z^{2}-\frac{1}{2} z^{3}=0.046537$

\begin{tabular}{|c|c|}
\hline $\mathrm{x}$ & 0 \\
$1-\mathrm{y}$ & 0 \\
0 & $\mathrm{x}$ \\
1 & $\mathrm{y}$ \\
$1-\mathrm{z}$ & $\mathrm{z}$ \\
$\mathrm{z}$ & $1-\mathrm{z}$ \\
0 & $1-\mathrm{y}$ \\
1 & $1-\mathrm{x}$ \\
$\mathrm{y}$ & 1 \\
$1-\mathrm{x}$ & 1 \\
\hline
\end{tabular}

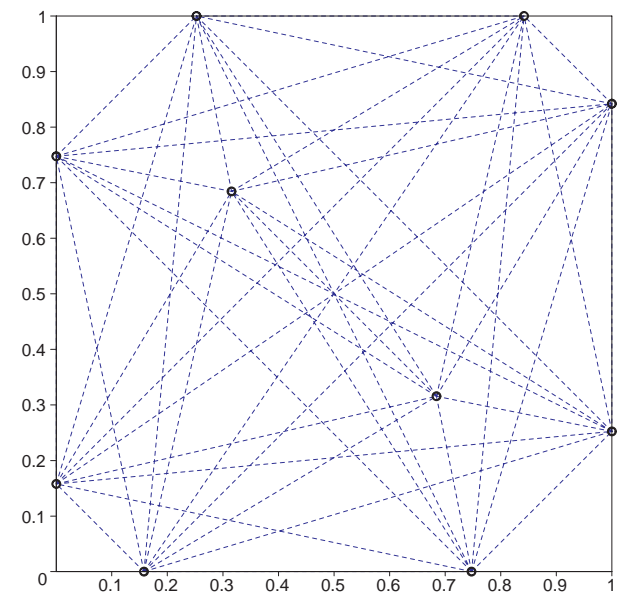




\section{Eleven points}

$H_{11} \geq \frac{1}{27}=0.037037$ (this result was already known)

\begin{tabular}{|c|c|}
\hline $1 / 3$ & 0 \\
$2 / 3$ & 0 \\
0 & $2 / 9$ \\
1 & $2 / 9$ \\
$1 / 3$ & $4 / 9$ \\
$2 / 3$ & $4 / 9$ \\
0 & $2 / 3$ \\
1 & $2 / 3$ \\
$1 / 2$ & $7 / 9$ \\
$1 / 6$ & 1 \\
$5 / 6$ & 1 \\
\hline
\end{tabular}

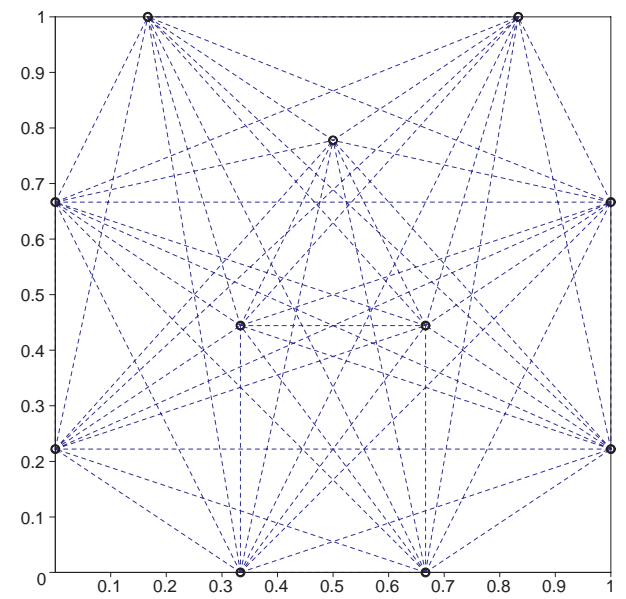

\section{Twelve points}

With $x=1-\frac{1}{6} \frac{(27+3 \sqrt{57})^{2 / 3}+6}{(27+3 \sqrt{57})^{1 / 3}}=0.115354$ and $y=2 x^{2}-3 x+1 / 2=0.180552$,

$H_{12} \geq \frac{1}{4} x+\frac{1}{2} x y-\frac{1}{2} x^{2}=0.032599$

\begin{tabular}{|c|c|}
\hline $\mathrm{x}$ & 0 \\
$1-\mathrm{x}$ & 0 \\
0 & $\mathrm{x}$ \\
1 & $\mathrm{x}$ \\
$\frac{1}{2}$ & $\mathrm{y}$ \\
$\mathrm{y}$ & $\frac{1}{2}$ \\
$1-\mathrm{y}$ & $\frac{1}{2}$ \\
$\frac{1}{2}$ & $1-\mathrm{y}$ \\
0 & $1-\mathrm{x}$ \\
1 & $1-\mathrm{x}$ \\
$\mathrm{x}$ & 1 \\
$1-\mathrm{x}$ & 1 \\
\hline
\end{tabular}

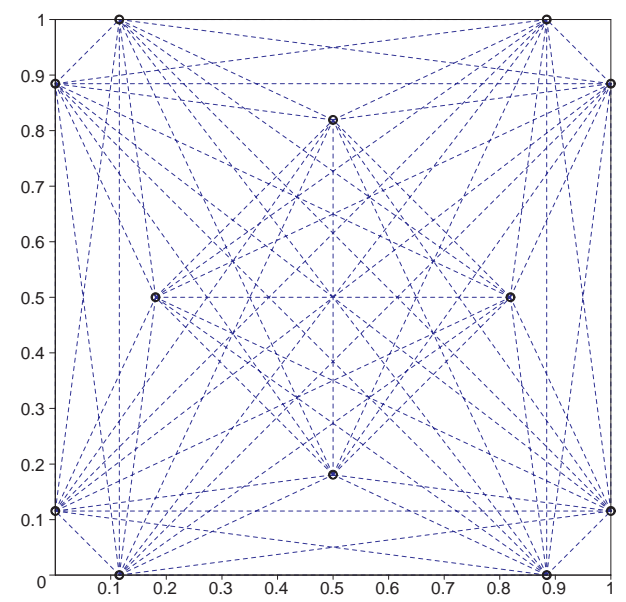




\section{Conclusion}

Combinatorial optimization techniques, like simulated annealing, can help to obtain first approximations that may be further refined analytically. They can provide insights to find unexpected configurations. In Figure 1 we present, as an example, the configuration given by Goldberg in [7], which provided the lower bound $H_{8} \geq \frac{2-\sqrt{3}}{4}=0.066987$ and the configuration produced using simulated annealing and a further analytical refinement, which leads to the new bound $H_{8} \geq \frac{\sqrt{13}-1}{36}=0.072376$.
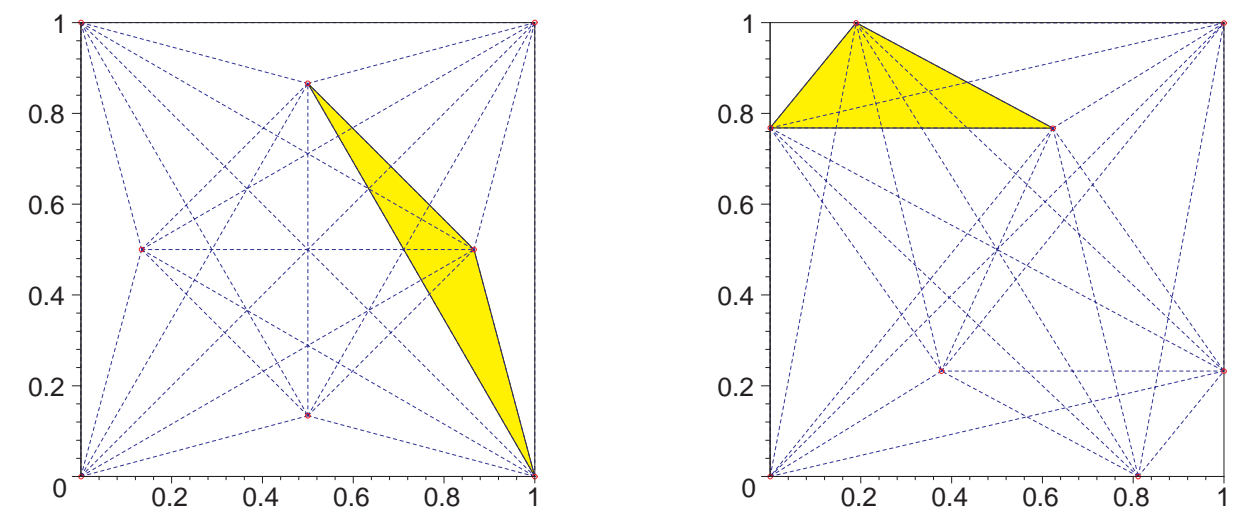

Figure 1: A former configuration leading to $H_{8} \geq \frac{2-\sqrt{3}}{4}=0.066987$ and a configuration found with simulated annealing that gives the new lower bound $H_{8} \geq \frac{\sqrt{13}-1}{36}=0.072376$.

Finally, the new bounds obtained are summarized in Table 1.

\begin{tabular}{|c||c|c|}
\hline & former bound & new bound \\
\hline$H_{5}$ & 0.192450 & \\
$H_{6}$ & 0.125000 & \\
$H_{7}$ & 0.083333 & 0.083859 \\
$H_{8}$ & 0.066987 & 0.072376 \\
$H_{9}$ & 0.047619 & 0.054876 \\
$H_{10}$ & 0.042791 & 0.046537 \\
$H_{11}$ & 0.037037 & \\
$H_{12}$ & 0.030303 & 0.032599 \\
\hline
\end{tabular}

Table 1: New Heilbronn numbers. 


\section{References}

[1] E. Aarts and J.K. Lenstra (eds.), Local Search in Combinatorial Optimization, John Wiley and Sons, Chichester, UK, 1997.

[2] G. Barequet, A lower bound for for Heilbronn's triangle problem in d dimensions. SIAM J. Discrete Math. 14 (2001), no. 2, 230-236.

[3] C. Bertram-Kretzberg, T. Hofmeister, and H. Lefmann, An algorithm for Heilbronn's problem, SIAM J. Comput. 30 (2000), no. 2, 383-390 .

[4] S. Carlson, Algorithm of the Gods, Scientific American 276 (1977), no. 3, 121-123.

[5] F. Comellas and J.L.A. Yebra, An optimization problem, manuscript.

[6] A.W.M. Dress, Yang Lu, and Zeng Zhenbing, Heilbronn problem for six points in a planar convex body. Combinatorics and Graph Theory'95. vol 1 (Hefei), 97-118, World Sci. Publishing, River Edge, N.J., 1995.

[7] M. Goldberg, Maximizing the smallest triangle made by N points in a square, Math. Magazine 45 (1972), 135-144.

[8] T. Jiang, M. Li, and P. Vitányi, The expected size of Heilbronn's triangles, Proceedings of the Fourteenth Annual IEEE Conference on Computational Complexity. 4-6 May 1999, Atlanta, Georgia, USA, 105-113

[9] T. Jiang, M. Li, and P. Vitányi, The average-case area of Heilbronn-type triangles, Random Struct. Alg., to appear.

[10] S. Kirkpatrick, C.D. Gelatt, and M.P. Vecchi, Optimization by Simulated Annealing. Science 220 (1983), 671-680.

[11] J. Komlós, J. Pintz, and E. Szemerédi, On Heilbronn's triangle problem, J. London Math. Soc. 24 (1981), no. 2, 385-396.

[12] J. Komlós, J. Pintz and E. Szemerédi, A lower bound for Heilbronn's problem, J. London Math. Soc. 25 (1982), n0. 2, 13-24.

[13] N. Metropolis, A. Rosenbluth, M. Rosenbluth, A. Teller, and E. Teller, J. Chem. Phys. 21 (1953), 1087-1092.

[14] K.F. Roth, On a problem of Heilbronn, J. London Math. Soc. 26 (1951), 198-204.

[15] K.F. Roth, On a problem of Heilbronn II, Proc. London Math Society (3) 25 (1972), 193-212.

[16] K.F. Roth, On a problem of Heilbronn III, Proc. London Math Society (3) 25 (1972), $543-549$. 
[17] K.F. Roth, Estimation of the area of the smallest triangle obtained by selecting three out of $n$ points in a disc of unit area. Proc. Symp. Pure Mathematics 24, AMS, Providence, 1973, 251-262.

[18] K.F. Roth, Developments in Heilbronn's triangle problem, Advances in Math. 22 (1976), 364-385 .

[19] W.M. Schmidt, On a problem of Heilbronn, J. London Math. Soc. (2) 4 (1972), $545-550$.

[20] Yang Lu, Zhang Jingzhong, and Zeng Zhenbing, On Goldberg's conjecture: Computing the first several Heilbronn numbers. Universität Bielefeld, Preprint 91-074 (1991).

[21] Yang Lu, Zhang Jingzhong, and Zeng Zhenbing, On exact values of Heilbronn numbers for triangular regions. Universität Bielefeld, Preprint 91-098 (1991).

[22] Yang Lu, Zhang Jingzhong, and Zeng Zhenbing, A conjecture on the first several Heilbronn numbers and a computation, Chinese Ann. Math. Ser. A 13 (1992), 503515. 